\title{
Controllability analysis of decentralised linear controllers for polymeric fuel cells
}

\author{
Maria Serra*, Joaquín Aguado, Xavier Ansede, Jordi Riera \\ Institut de Robòtica i Informàtica Industrial, Universitat Politècnica de Catalunya - Consejo Superior de Investigaciones Científicas, \\ C. Llorens i Artigas 4, 08028 Barcelona, Spain
}

\begin{abstract}
This work deals with the control of polymeric fuel cells. It includes a linear analysis of the system at different operating points, the comparison and selection of different control structures, and the validation of the controlled system by simulation. The work is based on a complex non linear model which has been linearised at several operating points. The linear analysis tools used are the Morari resiliency index, the condition number, and the relative gain array. These techniques are employed to compare the controllability of the system with different control structures and at different operating conditions. According to the results, the most promising control structures are selected and their performance with PI based diagonal controllers is evaluated through simulations with the complete non linear model. The range of operability of the examined control structures is compared. Conclusions indicate good performance of several diagonal linear controllers. However, very few have a wide operability range.
\end{abstract}

Keywords: Polymeric fuel cell; Diagonal control; Linear control; PI controllers; Operating conditions; Linear analysis

\section{Introduction}

Proton exchange membrane fuel cells (PEMFC), also known as polymer electrolyte fuel cells, are being actively developed for use in cars, buses, as well as for a very wide range of portable applications, and also for combined heat and power systems $[1,2]$. They are regarded as ideally suited for transportation applications due to its high power density, compactness, lightweight, low-operating temperature which permits fast start-up, solid electrolyte, long cell and stack live, low corrosion, and higher efficiencies compared to heat engines $[3,4]$.

A lot of works are dedicated to model polymeric fuel cells, as summarised by Yao et al. [5]. However, most of these models are static models. Some authors introduce electrochemical time constants in their models [6], but they do not model the flow dynamics of the whole system. With

\footnotetext{
* Corresponding author. Tel.: +34 934015805; fax: +34 934015750.

E-mail address: maserra@iri.upc.es (M. Serra).
}

the aim to study the flow dynamics of polymeric fuel cells, Pukrushpan et al. [7] presented a control oriented model which includes the transient phenomena of the compressor, the manifold filling dynamics (both anode and cathode), reactant partial pressures, and membrane humidity. This model proposed by Pukrushpan et al. has been the base for the model used in this work although some modifications are introduced.

In 1998, Yang et al. [8] described the control challenges in fuel cell vehicle development. After that, some studies have addressed these challenges related to the control of polymeric fuel cells $[4,9]$. This literature has been considered to define the multiple objectives of the control problem addressed in this work.

PI based controllers are proposed by some authors but a complete study of the multiple input multiple output (MIMO) control problem is not done. In this work, different control structures are analysed and compared taking into account the interactions between loops and the directionality of the system. 


\begin{tabular}{ll} 
Nomenclature \\
$A_{\mathrm{fc}}$ & active area per cell \\
$c_{\mathrm{V}}$ & water concentration \\
$I_{\mathrm{st}}$ & stack load current \\
$i$ & current density \\
$K_{\mathrm{sm}, \text { out }}$ & fluidynamic constant at supply manifold out- \\
& put \\
$K_{\mathrm{ca}, \text { out }}$ & fluidynamic constant at the cathode output \\
$K_{\mathrm{rm}, \text { out }}$ & fluidynamic constant at the return manifold \\
& output \\
$K_{\mathrm{an}, \text { out }}$ & fluidynamic constant at the anode output \\
$m_{\mathrm{i}}$ & mass of component i \\
$n$ & number of fuel cells \\
$p_{\mathrm{ca}}$ & cathode pressure \\
$p_{\mathrm{O}_{2}}$ & cathode oxygen partial pressure \\
$p_{\mathrm{H}}$ & anode hydrogen partial pressure \\
$p_{\mathrm{sat}}$ & saturation pressure of water at $T_{f c}$ \\
$\Delta p$ & anode-cathode pressure difference \\
$T_{\mathrm{fc}}$ & fuel cell temperature \\
$t_{\mathrm{m}}$ & membrane thickness \\
$v_{\mathrm{fc}}$ & unique fuel cell voltage \\
$v_{\mathrm{cm}}$ & the compressor voltage \\
$v_{\mathrm{st}}$ & stack voltage \\
$W_{\mathrm{i}}$ & mass flowrate of component i \\
$x_{\mathrm{H}_{2}, \text { an,in }}$ & the mass fraction of the hydrogen \\
$y_{\mathrm{O}_{2}, \text { ca,in }}$ & the molar fraction of input air \\
$\mathrm{Gree}_{\mathrm{m}}$ & letters \\
$\phi_{\mathrm{ca}, \text { in }}$ & the relative humidity of the input air \\
$\lambda_{\mathrm{O}_{2}}$ & oxygen utilisation \\
$\lambda_{\mathrm{m}}$ & membrane water content \\
\hline
\end{tabular}

To use fuel cells in specific applications, electric conditioning systems are normally added. The complexity and performance of these power electronic systems vary and consequently the stress suffered by the fuel cell varies too. Most literature works analyse the ability of the fuel cell control system to follow changes in the current load, and assume large current excursions. However, thanks to the conditioning systems, these excursions can be minimised, and the fuel cell maintained close to a nominal operating point. In this small region behaviour is closer to linear behaviour. In automotive applications, for example, fuel cells can be used in hybrid configurations with batteries allowing the cells to be operated at steady state with high-power output under optimised conditions, and with the peak power demands being met by batteries [10]. Alternatively, the fuel cells can be used as the sole power supply and sudden load application are a possible scenario. For this reason, in this work, large changes in the current load as well as small excursions from a nominal operating point are taken into account.

The rest of the paper is structured as follows: in Section 2 , the work methodology is described, Section 3 contains the description of the model, in Section 4 the control objectives are explained, in Section 5 the linear analysis is done and the preferred control structures are identified, in Section 6 the preferred control structures are validated through simulation, in Section 7 the best control possibilities are discussed, and finally, in Section 8 the main conclusions are explained. Two appendices recall some of the model equations and in the Nomenclature Section the different variables that appear in the work are defined.

\section{Methodology}

This work is based on a non linear model of a PEMFC proposed by Pukrushpan et al. [7], which has been linearised at different operating points. MIMO linear models are obtained in the form of state space matrices A, B, C, and D.

The work faces the control of two output variables using two of the free input variables. Therefore, $2 \times 2$ control structures are considered. In this work, a control structure is understood as the set of inputs chosen to control the system, as well as the input-output pairing. Since there exist more than two possible manipulated variables, there exist different pairs of input variables to control the system. From a linear system that includes all the input variables, the different $2 \times 2$ linear systems corresponding to the different input pairs are derived removing, in each case, the columns of the rejected inputs.

MIMO linear systems can be analysed using different analysis tools. These tools are mathematic operators applied to the squared transfer functions of the linear system that give relevant information such as stability, controllability, sensitivity, robustness, etc. To design the control of MIMO systems, one of the most important tasks is the selection of the set of manipulated variables to control the system. Different controllability indexes can help the designer choose this variables set.

In the literature, three different controllability indexes are frequently used [11]: the Morari resiliency index (MRI), the condition number $(\mathrm{CN})$, and the relative gain array (RGA). These indexes are used in this work to study the interaction between control loops and the sensitivity of the controlled system.

The singular value decomposition is a numerical algorithm useful in analysing the multivariable aspect of the gain matrix, giving the input and output directions for which gains are maximum and minimum. The MRI is the smallest singular value of the open-loop transfer function. It is the poorer gain of the process, poorer sensitivity, which corresponds to specific input and output directions. MRI is one of the controllability indexes calculated and analysed in this work. Control structures with large MRI over the frequency range of interest are preferred.

The second controllability index analysed is the CN. It also comes from the singular value decomposition of the transfer function. The $\mathrm{CN}$ is the ratio of the maximum singular value 
to the minimum singular value and it is typically used for the control structure selection. It provides a numerical indication of the sensitivity balance in a multivariable system. Large condition numbers indicate unbalanced sensitivity and also sensitivity to changes in process parameters. Therefore, structures with small CNs are preferred.

The third index analysed is the RGA. RGA is used to determine the interaction among control loops in a multivariable process. It is defined as the ratio of the open-loop gain for a selected output when all the other loops of the process are open, to its open-loop gain when all the other loops are closed. RGA of a complex non-singular matrix $\mathbf{M}$ is calculated as indicated in Eq. (1), where $\mathrm{X}$ denotes element by element multiplication (Hadamard product).

$\operatorname{RGA}(\mathbf{M})=\mathbf{M X}\left(\mathbf{M}^{-1}\right)^{\mathrm{T}}$

Pairings that have RGA close to unity matrix at frequencies around bandwidth are preferred. This rule favours minimal interaction between loops, which means independence of the loops. Being the loops independent, stability problems caused by interaction are prevented. Numbers around 0.5 indicate relevant interaction. The RGA indicates other useful control properties [11]. One of the most important, structures with large RGA elements around the bandwidth frequency are difficult to control because of sensitivity to input uncertainty.

In this work, these three indexes are used to characterise the controllability of polymeric fuel cells. They are used to compare different control structures for a PEMFC and a control structure at different operating conditions.

Normally, only the steady state value of these controllability indexes is regarded. However, their analysis in the frequency domain is important [11]. Consequently, in this work, the three indexes are analysed in a wide frequency range. The frequency range of interest is given by the bandwidth frequency. The bandwidth frequency is normally defined as the frequency up to which control is effective and it depends on the controller.

The main goal of this work is the linear analysis of the system. However, the PEMFC is not linear. Linearised model behaviour cannot be extrapolated when the system makes large excursions from the nominal operating point. To prove the capability of the preferred control structures to drive the system correctly, simulations based on a non linear system are necessary. Because of that, after the controllability analysis in Section 5, an analysis based on the non linear model simulations is done (Section 6).

\section{Model}

\subsection{Non linear model}

Results of this work are based on a non linear model proposed by Pukrushpan et al. [7]. The model was developed specifically for control with the idea of avoiding unnecessary detail. On the other hand, special attention is given to the air circuit subsystem and a quite accurate compressor model is included.

Important simplifications are done because for control purposes, excessive detail is not necessary. Because of that, spatial variations are not included and constant properties are assumed in all volumes. Only time derivatives are present. With respect to the considered dynamics, the model neglects the fast dynamics of electrochemical reactions and electrode electricity. Temperature is treated as a constant parameter because its slow behaviour (time constant of about $102 \mathrm{~s}$ ) permits that it is regulated with its own (slower) controller.

The fuel stack model contains four interacting parts: the stack voltage, membrane hydration, the cathode, and the anode.

Stack voltage, $v_{\mathrm{st}}$, is calculated as a function of stack current, $I_{\mathrm{st}}$, cathode pressure, $p_{\mathrm{ca}}$, reactant partial pressures, $p_{\mathrm{O}_{2}}$ and $p_{\mathrm{H}_{2}}$, fuel cell temperature, $T_{\mathrm{fc}}$, and membrane humidity. Identical behaviour of each cell is assumed and stack voltage is calculated as the individual cell voltage, $v_{\mathrm{fc}}$, per the number of cells, $n$. The cell voltage has four terms, as can be seen in the following equation

$v_{\mathrm{fc}}=E-v_{\mathrm{act}}-v_{\mathrm{ohm}}-v_{\mathrm{conc}}$

where $E$, the open circuit voltage, is a function of the fuel cell temperature and hydrogen and oxygen partial pressures; $v_{\text {act }}$, the activation overvoltage, depends on the current, the temperature, and the oxygen partial pressure; $v_{\mathrm{ohm}}$, the ohmic overvoltage, is proportional to the stack current and has a proportionality constant strongly dependent on membrane humidity and temperature; and $v_{\text {conc }}$, the concentration overvoltage, depends on the current and oxygen partial pressure. In Appendix A, detailed expressions are shown.

Membrane hydration captures the effect of water transport across the membrane. Both water content and mass flow are assumed to be uniform over the surface area of the membrane, and are functions of stack current and relative humidity of the gas in the anode and cathode. Detailed hydration model is given in Appendix B.

Inside the cathode volume, the mass flow continuity is used to balance the mass of the three elements (oxygen, nitrogen, and water). Some assumptions are made: ideal gases, cathode temperature equal to the stack temperature, same properties of the gas exiting the cathode and the gas inside the cathode, and flow channels and backing layer lumped into one volume. Equations for the mass time derivatives are:

$\frac{\mathrm{d} m_{\mathrm{O}_{2}}}{\mathrm{~d} t}=W_{\mathrm{O}_{2}, \text { in }}-W_{\mathrm{O}_{2}, \text { out }}-W_{\mathrm{O}_{2}, \text { reacted }}$

$\frac{\mathrm{d} m_{\mathrm{N}_{2}}}{\mathrm{~d} t}=W_{\mathrm{N}_{2}, \text { in }}-W_{\mathrm{N}_{2}, \text { out }}$

$\frac{\mathrm{d} m_{\mathrm{w}, \mathrm{ca}}}{\mathrm{d} t}=W_{\mathrm{v}, \mathrm{ca}, \text { in }}-W_{\mathrm{v}, \mathrm{ca}, \text { out }}+W_{\mathrm{v}, \mathrm{ca}, \text { gen }}+W_{\mathrm{v}, \mathrm{membr}}$

$W_{\mathrm{O}_{2}, \text { reacted }}=M_{\mathrm{O}_{2}} \times \frac{n I_{\mathrm{st}}}{4 F}$ 
$W_{\mathrm{v}, \mathrm{ca}, \mathrm{gen}}=M_{\mathrm{v}} \times \frac{n I_{\mathrm{st}}}{2 F}$

where $W_{\text {in }}$ and $W_{\text {out }}$ are the flow rates entering and exiting the

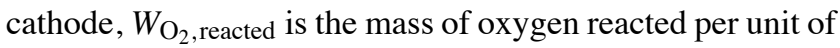
time, $W_{\mathrm{v}, \mathrm{ca} \text {, gen }}$ is the mass of water generated by the reaction per unit of time, $W_{\mathrm{v}, \mathrm{membr}}$ is the mass of water that crosses the membrane per unit of time, $M_{\mathrm{O}_{2}}$ and $M_{\mathrm{v}}$ the molar masses of oxygen and water, and $F$ the Faraday constant.

The air entering the cathode is impelled by a compressor. The modelled system includes a compressor with a dynamic part and a static part the behaviour of which is read from an experimental compressor map. Supply and return manifolds, cooler and humidifier are also included. Supply and return manifolds are lumped and modelled as unique volumes. The supply manifold pressure is governed by mass continuity and energy conservation equations assuming ideal gas. The return manifold pressure is governed by the mass continuity and the ideal gas law through isothermal assumptions. An ideal cooler maintains the temperature of the air entering the stack at a fixed point. The pressure drop across the cooler is negligible. It is assumed that a static humidifier gives to the air the desired relative humidity before entering the stack and the amount of water injected is calculated.

Flow rates from one volume to another are calculated in function of the upstream and downstream pressures. The same linear behaviour is assumed at the supply manifold output, cathode output, and return manifold output. In all cases, the following expression applies, where $p_{\text {upstream }}$ is the upstream pressure and $p_{\text {downstream }}$ is the downstream pressure:

$W=K_{\text {nozzle }} \times\left(p_{\text {upstream }}-p_{\text {downstream }}\right)$.

At the anode side, entering hydrogen comes from a pressurised tank. Since pressurised hydrogen is used, the hydrogen flow is assumed to be a manipulated input variable. Although the model used in this work is based on the model presented by Pukrushpan et al. [7], an important modification is done: the addition of an exit flow in the anode. This is necessary to keep the system flooded with gas and to improve the power demand transient responses [8]. To model this flow, a linear behaviour as indicated in Eq. (8) is assumed.

The anode flow model is quite similar to the cathode flow model. Hydrogen partial pressure and anode flow humidity are determined by balancing the mass of hydrogen and water in the anode.

$\frac{\mathrm{d} m_{\mathrm{H}_{2}}}{\mathrm{~d} t}=W_{\mathrm{H}_{2}, \text { in }}-W_{\mathrm{H}_{2}, \text { out }}-W_{\mathrm{H}_{2}, \text { reacted }}$
$\frac{\mathrm{d} m_{\mathrm{w}, \text { an }}}{\mathrm{d} t}=W_{\mathrm{v}, \text { an }, \text { in }}-W_{\mathrm{v}, \text { an, out }}-W_{\mathrm{v}, \text { membr }}$

where $W_{\text {in }}$ and $W_{\text {out }}$ are the flow rates entering and exiting the anode and $W_{\mathrm{H}_{2}}$,reacted is the mass of hydrogen reacted per unit of time.

All together, it results in a model of nine states: the angular velocity of the compressor, the mass of oxygen in the cathode, the mass of nitrogen in the cathode, the mass of water vapour in the cathode (saturated for some operating conditions), the mass of hydrogen in the anode, the mass of water vapour in the anode, the pressure in the supply manifold, the mass of air in the supply manifold, and the pressure in the return manifold. In case of water saturation, eight states are contemplated instead of nine because the mass of water vapour in the cathode is removed. More detail of the non linear model can be found in [7].

\subsection{Model linearisation}

The non linear model described above has been linearised at different operating points. SIMULINK ${ }^{\circledR}$ linearisation tools have been used to obtain the state space matrices of the system.

MRI and $\mathrm{CN}$ are scale dependent. Because of that, the linear models have been scaled. One of the controlled outputs is the difference of pressure between anode and cathode, $\Delta p$, with a maximum accepted variation of 0.1 bar. This has been used as the scaling parameter. For the rest of input and output variables, scaling has been done assuming a maximum variation of $10 \%$. Hence, the scaled variables are the non scaled increments divided by the maximum increments.

\subsection{Operating conditions}

The modelled fuel cell has a number of cells $n=381$, an active area per cell $A_{\mathrm{fc}}=280 \mathrm{~cm}^{2}$, and an operating temperature $T_{\mathrm{fc}}=80^{\circ} \mathrm{C}$. Linearisation of the system has been done at four different operating points that have been called OP1, $\mathrm{OP} 2, \mathrm{OP} 3$, and OP4. In all cases, the parameters of the model predict full humidified cathode and the water vapour in the cathode is saturated [7].

For OP1, a net power of $P_{\text {net }}=37,400 \mathrm{~W}$ is required. The net power is the power given by the fuel cell minus the power consumed by the compressor. The desired amount of power can be obtained at different load currents. However, there is a minimum load current for which this power can be obtained, and its value is $I_{\mathrm{st}}=175 \mathrm{~A}$. With the aim of having OP1 with the minimum hydrogen consumption (minimum hydrogen reaction if hydrogen is recycled), an operating point with $I_{\mathrm{st}}=175 \mathrm{~A}$ is chosen. $\mathrm{OP} 1$ is then defined by $I_{\mathrm{st}}=175 \mathrm{~A}$, $v_{\mathrm{cm}}=158 \mathrm{~V}, W_{\mathrm{an}, \mathrm{in}}=1.0134 \mathrm{~g} \mathrm{~s}^{-1}$, giving an output stack voltage of $v_{\mathrm{st}}=242.75 \mathrm{~V}, p_{\mathrm{ca}}=1.99 \mathrm{bar}, \Delta p=-0.0068 \mathrm{bar}$, and $P_{\text {net }}=37,400 \mathrm{~W}$. With these conditions the system is operating in the linear zone of the polarisation curve, with a current density $i=0.62 \mathrm{~A} \mathrm{~cm}^{-2}$.

OP2 and OP3 have the same output voltage than OP1 and the same $\Delta p$, but different current loads. OP2 is defined by $I_{\mathrm{st}}=150 \mathrm{~A}$ (closer to the activation zone), and has $v_{\mathrm{cm}}=137 \mathrm{~V}, W_{\mathrm{an}, \mathrm{in}}=0.86 \mathrm{~g} \mathrm{~s}^{-1}$, and $p_{\mathrm{ca}}=1.82 \mathrm{bar}$; OP 3 is defined by $I_{\mathrm{st}}=200 \mathrm{~A}$ (closer to the concentration zone), and has $v_{\mathrm{cm}}=183 \mathrm{~V}, W_{\mathrm{an}, \text { in }}=1.18 \mathrm{~g} \mathrm{~s}^{-1}$, and $p_{\mathrm{ca}}=2.21$ bar. Therefore, OP2 and OP3 are operating points the controlled system will pass through with load currents of 150 and $200 \mathrm{~A}$.

Finally, OP4 is defined fixing the net power at $37,400 \mathrm{~W}$ and $I_{\mathrm{st}}=200 \mathrm{~A}$. The corresponding compressor voltage is 
$v_{\mathrm{cm}}=130.24 \mathrm{~V}, v_{\mathrm{st}}=201.85 \mathrm{~V}$, and $p_{\mathrm{ca}}=1.73$ bar. OP4 has the same net power as OP1 but a higher hydrogen consumption. It has been chosen to compare the controllability of the system when it is operated at optimal and non-optimal conditions with respect to the hydrogen consumption.

\section{Analysis of the control problem and control objectives}

The first control objective addressed in this work is to maintain $v_{\text {st }}$ to the setpoint value. On the other hand, in order to prevent membrane damage, the difference of pressure between anode and cathode $\Delta p$ has to be small [8]. The adequate $\Delta p$ value will depend on the membrane support and on the age of the fuel cell. However, since minimum $\Delta p$ will favour the membrane life time, the second control objective addressed is to maintain $\Delta p$ close to zero.

The inputs of the system are the oxygen molar fraction of the dry input air, $y_{\mathrm{O}_{2} \text {,ca,in }}$, the compressor voltage, $v_{\mathrm{cm}}$, the flow of hydrogen $W_{\text {an,in }}$, the relative humidity of the input air, $\phi_{\mathrm{ca}, \text { in }}$, the mass fraction of the hydrogen (hydrogen

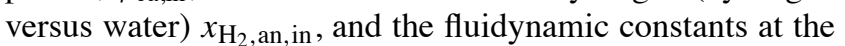
supply manifold output, cathode output, return manifold output, and anode output, $K_{\text {sm,out }}, K_{\text {ca,out }}, K_{\text {rm,out }}$, and $K_{\text {an,out }}$, respectively. Some of these inputs may not be manipulated variables in real applications, as will be discussed in Section 7. However, their inclusion in the controllability analysis may give important information about their influence.

As has been explained, there are two output variables to be controlled and nine different inputs. In consequence, there are multiple control structures which are possible. Characterisation and comparison between them is done in Sections 5 and 6 .

In the literature, the importance of the control of oxygen excess ratio $\lambda_{\mathrm{O}_{2}}$ is stressed [4]. Abrupt changes of this variable should be avoided in order to prevent abrupt changes in the oxygen partial pressure and oxygen starvation. The control of $\lambda_{\mathrm{O}_{2}}$ instead of the control of $v_{\mathrm{st}}$ is also studied in Section 6.

From this point on, control structures are designed by a pair of control variables, and it is understood that the first of them controls $v_{\text {st }}$ and the second of them controls $\Delta p$.

\section{Results of the linear analysis}

The results of the analysis of MRI, CN and RGA in the frequency range between $10^{-2}$ and $10^{2} \mathrm{rad} \mathrm{s}^{-1}$ are summarised in this section. Frequencies larger than $10^{2} \mathrm{rad} \mathrm{s}^{-1}$ are not analysed because it is considered that it is not convenient for the system to follow such rapid frequencies and, therefore, bandwidth frequency will not be larger than $10^{2} \mathrm{rad} \mathrm{s}^{-1}$.

Since two manipulated variables have to be selected from nine possibilities, there are 36 possible sets, or control structures. All 36 sets have been compared using the three indexes

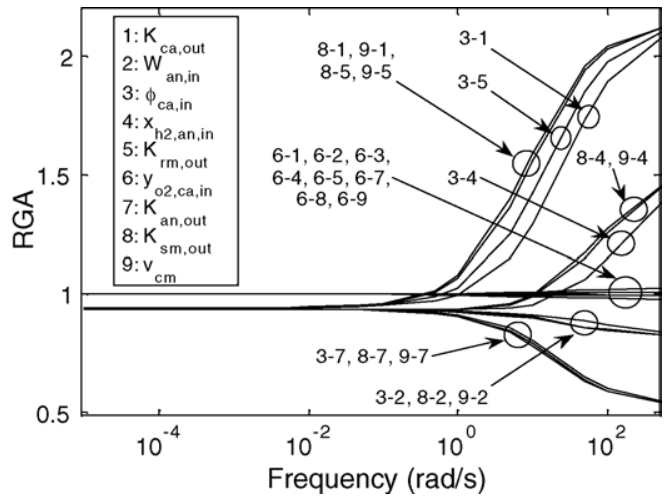

Fig. 1. RGA at operating point one.

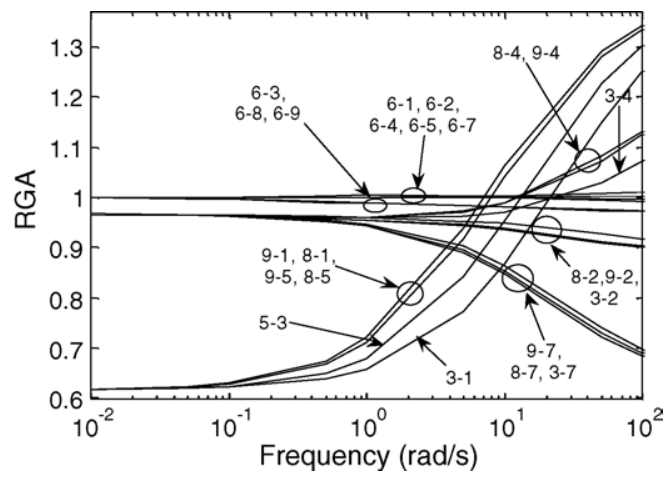

Fig. 2. RGA at operating point four.

in the frequency domain. However, in Figs. 1-6, only the indexes with best behaviours are plotted.

One property of the RGA is that the sum of all the elements of a row or a column is one [11]. In the case of two dimension RGAs, knowing one of the four elements of the matrix, the others are quickly known. Because of that, only the $(1,1)$ element is plotted (Figs. 1 and 2). As has been explained, diagonal RGA $(\operatorname{RGA}(1,1)=1)$ are preferred. $C N$ and MRI do not depend on the control structure pairing and in Figs. 3-6, the control structure names are indicated regardless of the order.

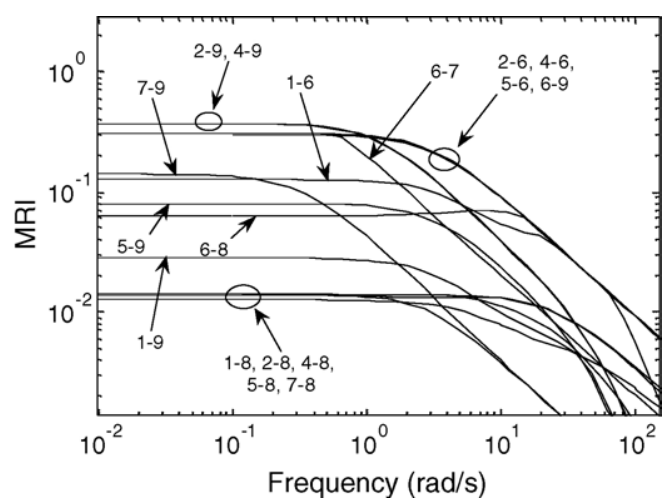

Fig. 3. MRI at operating point one. 


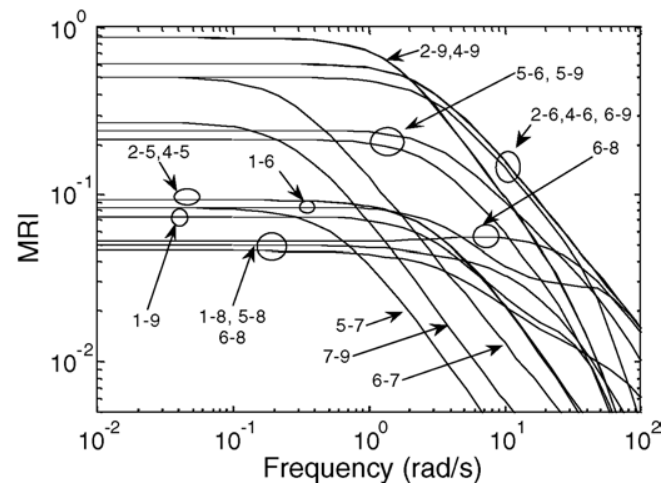

Fig. 4. MRI at operating point four.

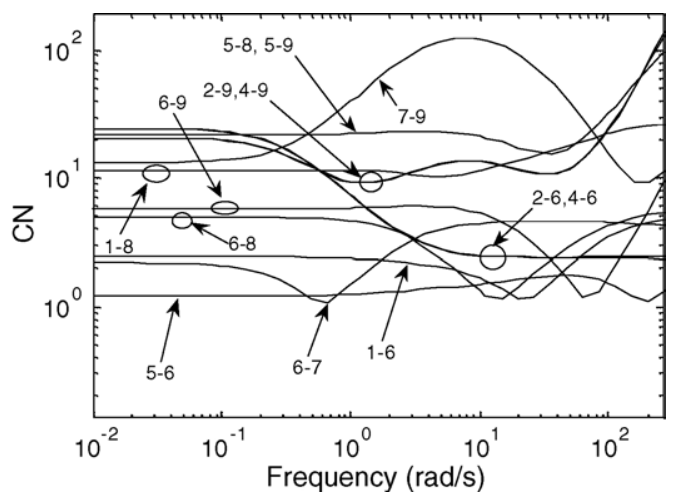

Fig. 5. $\mathrm{CN}$ at operating point one.

\subsection{Analysis at $O P 1$}

(1) In Fig. 1, only 23 of the 36 control structures are represented. Absent structures are the ones with the worst behaviour.

(2) All control structures with the variable $y_{\mathrm{O}_{2}}$,ca,in in the set of manipulated variables have $\operatorname{RGA}(1,1)$ very close to one, indicating a decoupled multivariable system. The pairing in all cases is such that $y_{\mathrm{O}_{2}}$,ca,in is in charge of $v_{\text {st }}$ control. This superior behaviour is independent of the frequency.

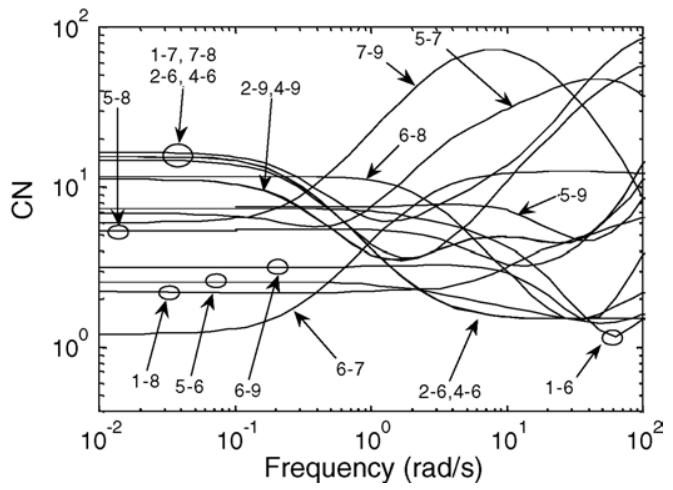

Fig. 6. $\mathrm{CN}$ at operating point four.
(3) Three control structures with $W_{\text {an,in }}$ controlling $\Delta p$ also have a $\operatorname{RGA}(1,1)$ very close to one. They are $\left(\phi_{\mathrm{ca}, \text { in }}-W_{\mathrm{an}, \text { in }}\right),\left(K_{\mathrm{sm}, \text { out }}-W_{\mathrm{an}, \text { in }}\right)$, and $\left(v_{\mathrm{cm}}-W_{\mathrm{an}, \text { in }}\right)$. The next group of structures with well behaved RGA $(1,1)$ are $\left(\phi_{\mathrm{ca}, \text { in }}-x_{\mathrm{H}_{2} \text {,an,in }}\right),\left(K_{\mathrm{sm}, \text { out }}-x_{\mathrm{H}_{2}, \text { an, in }}\right)$, and $\left(v_{\mathrm{cm}}-x_{\mathrm{H}_{2}, \text { an }, \text { in }}\right)$.

(4) In Fig. 3 , the best MRI behaviours are plotted. $\left(v_{\mathrm{cm}}-\right.$ $\left.W_{\mathrm{an}, \text { in }}\right)$ and $\left(v_{\mathrm{cm}}-x_{\mathrm{H}_{2} \text {,an,in }}\right)$ have the largest MRI of all the control structures at steady state and $\left(y_{\mathrm{O}_{2}, \mathrm{ca} \text {,in }}-\right.$ $\left.W_{\text {an,in }}\right),\left(y_{\mathrm{O}_{2}, \text { ca, in }}-x_{\mathrm{H}_{2} \text {, an,in }}\right),\left(y_{\mathrm{O}_{2}, \text { ca, in }}-K_{\mathrm{rm}, \text { out }}\right)$, and $\left(y_{\mathrm{O}_{2}, \mathrm{ca} \text {,in }}-v_{\mathrm{cm}}\right)$ have the largest MRI at high frequencies. It is remarkable that again, structures with the vari-

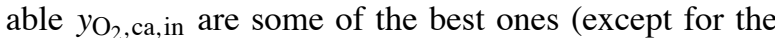
$\left.\left(y_{\mathrm{O}_{2}, \mathrm{ca}, \text { in }}-\phi_{\mathrm{ca}, \text { in }}\right)\right) .\left(v_{\mathrm{cm}}-K_{\mathrm{rm}, \text { out }}\right)$ has also a good behaviour.

(5) With respect to the $\mathrm{CN}$, the best control structure is $\left(y_{\mathrm{O}_{2}, \text { ca, in }}-K_{\mathrm{rm} \text {,out }}\right)$, followed by other structures with the variable $y_{\mathrm{O}_{2}, \text { ca,in }}:\left(y_{\mathrm{O}_{2}, \text { ca, in }}-K_{\mathrm{ca} \text {,out }}\right),\left(y_{\mathrm{O}_{2}, \text { ca,in }}-\right.$

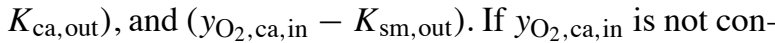
sidered, the best control structures with respect to the $\mathrm{CN}$ are $\left(K_{\mathrm{sm}, \text { out }}-K_{\mathrm{ca}, \text { out }}\right),\left(v_{\mathrm{cm}}-W_{\mathrm{an}, \text { in }}\right)$, and $\left(v_{\mathrm{cm}}-\right.$ $\left.x_{\mathrm{H}_{2} \text {,an,in }}\right)$. The next group of structures with well behaved $\mathrm{CN}$ are $\left(K_{\mathrm{sm}, \text { out }}-K_{\mathrm{rm}, \text { out }}\right)$ and $\left(v_{\mathrm{cm}}-K_{\mathrm{rm}, \text { out }}\right)$.

(6) Results show different behaviour of control structures with different location of fluidynamic restriction $\left(K_{\text {an,out }}\right.$, $K_{\text {ca,out }}, K_{\text {sm,out }}$, or $\left.K_{\text {rm,out }}\right)$. This indicates that controlling the system with one $K_{\text {nozzle }}$ or another has not the same effects. However, $K_{\text {ca,out }}$ and $K_{\text {rm,out }}$ will produce similar behaviour because there is only a small volume between these two fluid ways.

Considering the results of the three indexes, the following general conclusions for the controllability around OP1 are:

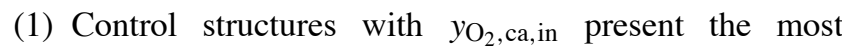
favourable indexes: RGA closest to unity matrix, highest MRI, and smallest CN.

(2) If $y_{\mathrm{O}_{2}}$,ca,in is not considered, the following best control structures are $\left(v_{\mathrm{cm}}-W_{\mathrm{an}, \text { in }}\right)$ and $\left(v_{\mathrm{cm}}-x_{\mathrm{H}_{2} \text {,an,in }}\right)$. These structures have very similar behaviour with respect to the controllability indexes. However, $x_{\mathrm{H}_{2} \text {, an, in }}$ should not be used as manipulated variable because of the delicate equilibrium of the water management system.

(3) other structures with good indexes are $\left(v_{\mathrm{cm}}-K_{\mathrm{rm} \text {,out }}\right)$, followed by $\left(v_{\mathrm{cm}}-K_{\mathrm{an}, \text { out }}\right)$. The last one has very good properties at low frequencies, but not at high frequencies.

\subsection{Analysis at $O P 2$ and $O P 3$}

Analysing the MRI and $\mathrm{CN}$ in the frequency domain at OP2 and OP3, some changes are observed with respect to the analysis at OP1. Therefore, the controllability indexes depend on the linearisation point. However, the structures presenting the higher MRI are the same as at OP1 $\left(\left(v_{\mathrm{cm}}-W_{\mathrm{an}, \mathrm{in}}\right)\right.$, $\left(v_{\mathrm{cm}}-x_{\mathrm{H}_{2}, \text { an,in }}\right),\left(y_{\mathrm{O}_{2}, \mathrm{ca}, \text { in }}-W_{\mathrm{an}, \text { in }}\right),\left(y_{\mathrm{O}_{2}, \mathrm{ca}, \text { in }}-x_{\mathrm{H}_{2}, \text { an,in }}\right)$, $\left.\left(y_{\mathrm{O}_{2}, \mathrm{ca}, \text { in }}-K_{\mathrm{rm}, \text { out }}\right), \quad\left(y_{\mathrm{O}_{2}, \mathrm{ca}, \text { in }}-v_{\mathrm{cm}}\right)\right)$, and the MRI 
values do not differ more than $10 \%$. The structures presenting smaller $\mathrm{CN}$ are also the same as at OP1. Only $\left(K_{\text {sm,out }}-K_{\text {ca,out }}\right)$ has a $\mathrm{CN}$ quite different in OP1, OP2, and OP3. Therefore, moving from OP1 to OP2 or OP3, the sensitivity of the system is almost unchanged for these preferred structures. Finally, RGA analysis indicates that some input pairs would require different pairings at OP2 and OP3. This is the case of the pair formed by $K_{\text {ca,out }}$ and $W_{\text {an,in. }}$ Evidently, these structures could not work properly around both operating points. However, the best structures according to RGA are the same at OP1, OP2, and OP3. As conclusion, when the system is controlled at constant $v_{\text {st }}$ and $\Delta p$, the preferred control structures will not change due to a variation in the operating conditions in a wide range of operation and the same tuning parameters are expected to be adequate for operation around OP1, OP2, and OP3.

\subsection{Analysis at $\mathrm{OP} 4$}

Analysing the MRI, CN and RGA in the frequency range between $10^{-2}$ and $10^{2} \mathrm{rad} \mathrm{s}^{-1}$ for the system linearised at OP4 (see Figs. 2, 4 and 6), the following can be stressed:

(1) The three general considerations done for the optimal operating point stand.

(2) Comparing results for OP1 and OP4, the most interesting structures $\left(\left(v_{\mathrm{cm}}-W_{\mathrm{an}, \mathrm{in}}\right),\left(v_{\mathrm{cm}}-x_{\mathrm{H}_{2}, \text { an,in }}\right),\left(v_{\mathrm{cm}}-\right.\right.$ $\left.K_{\text {an,out }}\right)$, and $\left.\left(v_{\mathrm{cm}}-K_{\text {rm,out }}\right)\right)$ have more than two times better indexes at all frequencies for OP4. This result is interesting because it indicates a possible trade off between optimal operation (minimal consumption) and controllability.

\section{Simulations}

Results of Section 5 are based on a linear model. The nonlinearities of the system are not considered. In order to study the nonlinearities influence, different simulations are done with the non linear model. PI controllers are implemented in the control loops. In Fig. 7, the response of the system to changes in $I_{\mathrm{st}}$ is shown. The $I_{\mathrm{st}}$ profile can be seen in Fig. $7 \mathrm{~h}$.

According to Section 5 results, the more interesting structures are some of the structures with $y_{\mathrm{O}_{2}, \mathrm{ca} \text {, in }}$, as well as $\left(v_{\mathrm{cm}}-W_{\mathrm{an}, \text { in }}\right),\left(v_{\mathrm{cm}}-x_{\mathrm{H}_{2}, \text { an ,in }}\right),\left(v_{\mathrm{cm}}-K_{\mathrm{rm}, \text { out }}\right)$ and $\left(v_{\mathrm{cm}}-K_{\text {an,out }}\right)$. From the most interesting structures with $y_{\mathrm{O}_{2} \text {, ca,in }}\left(y_{\mathrm{O}_{2}, \text { ca,in }}-W_{\text {an, in }}\right)$ has been chosen for simulation. On the other hand, structures implying variables related to the system humidity are rejected because their appropriate values have to be maintained in order to keep the delicate water equilibrium. In consequence, the structures chosen for the simulation comparison are $\left(y_{\mathrm{O}_{2}, \text { ca,in }}-W_{\mathrm{an}, \text { in }}\right),\left(v_{\mathrm{cm}}-W_{\mathrm{an}, \text { in }}\right)$, $\left(v_{\mathrm{cm}}-K_{\mathrm{rm} \text {,out }}\right)$, and $\left(v_{\mathrm{cm}}-K_{\mathrm{an}, \text { out }}\right)$.

Results of Section 5 indicate that $\left(y_{\mathrm{O}_{2}, \text { ca, in }}-W_{\text {an,in }}\right)$ and $\left(v_{\mathrm{cm}}-W_{\mathrm{an}, \text { in }}\right)$ have almost constant controllability indexes at OP1, OP2, and OP3. It has also been seen that
$\left(y_{\mathrm{O}_{2}, \text { ca,in }}-W_{\text {an,in }}\right)$ is less affected by the directionality of the system and has less influence between loops. As expected, simulations show that, with unique tuning parameters, both control structures are able to drive the system from one point to another with acceptable performances. Referring to the operating range limits, steps of $\pm 50 \%$ in the current load (not shown) are affordable for both structures with smooth tunings (if the $y_{\mathrm{O}_{2}}$, ca, in loop is tuned for an aggressive response, $y_{\mathrm{O}_{2}}$,ca,in can arrive to its physical limits). However, simulations have shown some differences between these two control structures, which have been tuned to have similar output peaks. Since the compressor dynamics only affects $\left(v_{\mathrm{cm}}-W_{\mathrm{an}, \mathrm{in}}\right)$, this structure is a bit slower, as can be appreciated in Fig. 7a and b. The total pressure of the cathode is much better maintained by $\left(y_{\mathrm{O}_{2}, \text { ca,in }}-W_{\text {an,in }}\right)$ and this provokes smaller changes in $W_{\text {an,in }}$, as can be seen in Fig. 7e. Another advantage of $\left(y_{\mathrm{O}_{2}}\right.$,ca,in $\left.-W_{\mathrm{an}, \text { in }}\right)$ that can be seen in Fig. $7 f$, is that this structure can maintain $\lambda_{\mathrm{O}_{2}}$ almost constant. In Fig. $7 \mathrm{~g}$, the net power is shown. With $\left(v_{\mathrm{cm}}-W_{\mathrm{an}, \mathrm{in}}\right)$ structure, the power consumed by the compressor is higher when the $I_{\mathrm{st}}$ is higher and lower when the $I_{\mathrm{st}}$ is lower and therefore, the net power increments and decrements are always diminished resulting in a more constant value. Since $\left(y_{\mathrm{O}_{2}}\right.$, ca,in $\left.-W_{\mathrm{an} \text {,in }}\right)$ does not modify $v_{\mathrm{cm}}$, the power consumed by the compressor is almost constant and the net power more variant with the $I_{\mathrm{st}}$ changes.

When analysing the control structures $\left(v_{\mathrm{cm}}-K_{\mathrm{an}, \mathrm{out}}\right)$ and $\left(v_{\mathrm{cm}}-K_{\mathrm{rm} \text {,out }}\right)$, very different behaviours are found close to the nominal operating point and far from this operating point. These structures are not able to control the system if the load current has large variations because of the hydrogen exhaustion. In Fig. $7 \mathrm{a}$ it is seen that $\left(v_{\mathrm{cm}}-K_{\mathrm{rm} \text {,out }}\right)$ can afford the first current step but is not able to control the system for the second current step. $\left(v_{\mathrm{cm}}-K_{\mathrm{an} \text {,out }}\right)$ has a wider range of operability than $\left(v_{\mathrm{cm}}-K_{\mathrm{rm} \text {,out }}\right)$ but its operability range is still much smaller than the operability range of $\left(y_{\mathrm{O}_{2}, \mathrm{ca}, \text { in }}-W_{\mathrm{an}, \text { in }}\right)$ and $\left(v_{\mathrm{cm}}-W_{\mathrm{an}, \text { in }}\right)$. Therefore, these two structures would work properly close to the nominal operating point but would not be able to drive the system for large excursions.

Simulation results show peaks in $\lambda_{\mathrm{O}_{2}}$ (Fig. 7f). These peaks are inadequate and can provoke oxygen starvation and the rapid degradation of the membrane. Through simulations which are not shown, $\lambda_{\mathrm{O}_{2}}$ behaviour has been compared in two different cases: the control of $v_{\mathrm{st}}$ by $v_{\mathrm{cm}}$ and the control of $\lambda_{\mathrm{O}_{2}}$ by $v_{\mathrm{cm}}$. Results indicate that similar $\lambda_{\mathrm{O}_{2}}$ peaks are obtained. Tuning parameters hardly influence the value of this peak. Therefore, the control of $\lambda_{\mathrm{O}_{2}}$ directly cannot solve the problem of the quick peak in $\lambda_{\mathrm{O}_{2}}$. In addition, the measure of $\lambda_{\mathrm{O}_{2}}$ would be difficult.

\section{Discussion}

Results of linear analysis indicate that control structures using $y_{\mathrm{O}_{2}, \mathrm{ca} \text {,in }}$ would be very adequate, and simulations 
with structure $\left(y_{\mathrm{O}_{2}, \mathrm{ca} \text {,in }}-W_{\mathrm{an}, \text { in }}\right)$ confirm its appropriate behaviour. However, manipulation of $y_{\mathrm{O}_{2}}$,ca, in would imply an added complexity $\left(\mathrm{N}_{2}\right.$ and $\mathrm{O}_{2}$ sources would be required). This added complexity will compensate or not the controllability improvement in dependence of the application.

As has been seen, when the fuel cell is operated close to a nominal operating point, control structures that use $K_{\text {an,out }}$
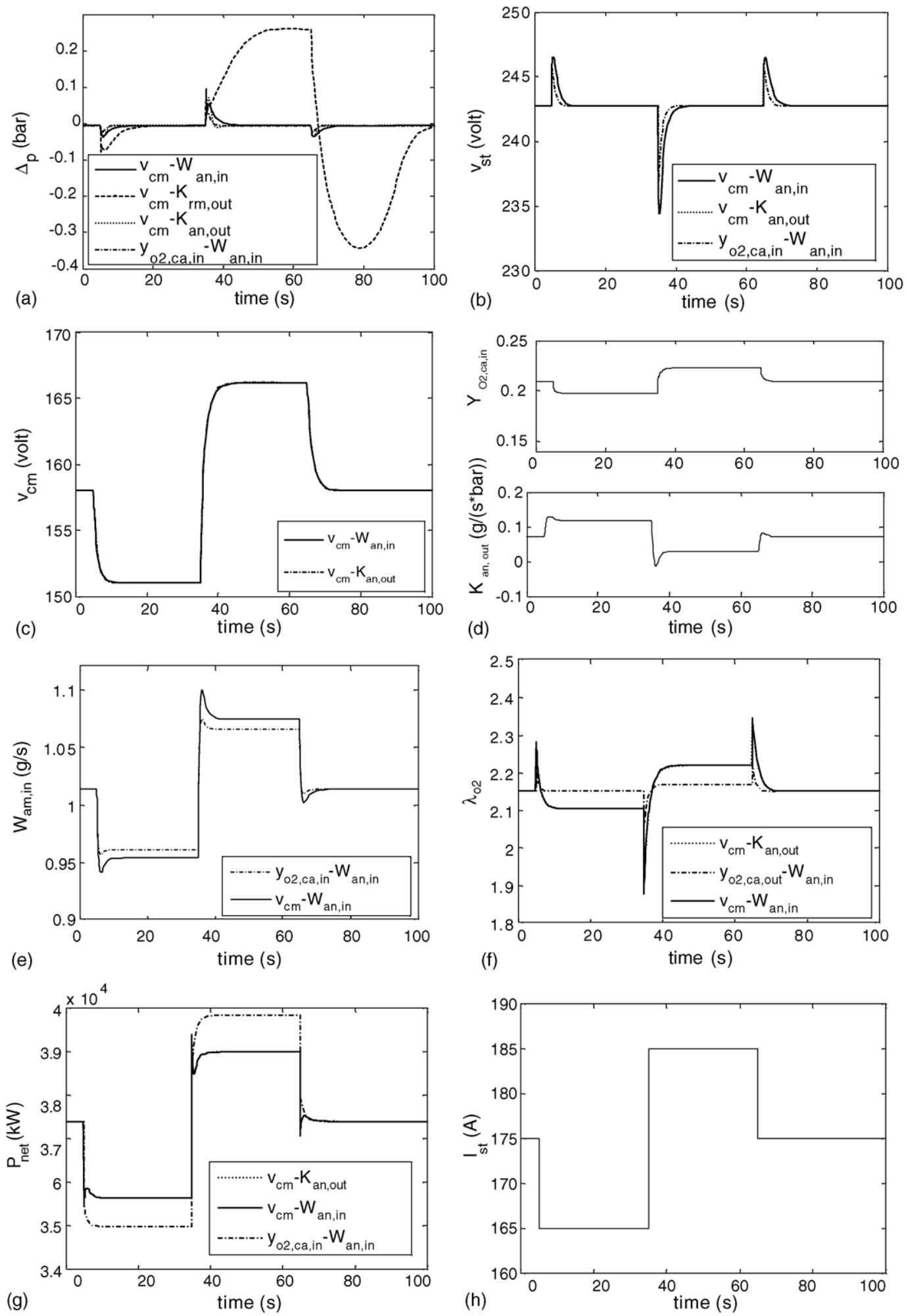

Fig. 7. Simulation results for $I_{\text {st }}$ variation shown in $\mathrm{h}$. 
are possible. Using this control variable, hydrogen output flow varies with $I_{\mathrm{st}}$. On the contrary, if $W_{\mathrm{an}, \text { in }}$ is used, hydrogen output flow is better maintained at the nominal value, what is important for a regular behaviour at the end of the distribution channels. $\left(v_{\mathrm{cm}}-W_{\mathrm{an} \text {, in }}\right)$ is clearly superior than $\left(v_{\mathrm{cm}}-K_{\mathrm{an}, \mathrm{out}}\right)$, but this second structure can still be useful in case of failure in the $\left(v_{\mathrm{cm}}-W_{\mathrm{an}, \text { in }}\right)$ control loop, for a correct shut down, for example. Close to a nominal operation, also $\left(v_{\mathrm{cm}}-K_{\mathrm{rm} \text {,out }}\right)$ is a valid structure. However, the limitations in this case are still more restrictive. Being both control variables in the cathode side, conflicts between loops appear easily.

A rapid control of $v_{\mathrm{st}}$ appears to be contradictory with slow variations of $p_{\mathrm{O}_{2}}$ because of the close relation between these two variables. Therefore, it seems that in order to avoid $p_{\mathrm{O}_{2}}$ peaks and oxygen starvation, $v_{\mathrm{st}}$ settling time must be relaxed. This limit of the fuel cell dynamic behaviour will influence the design of the conditioning system and its control objectives.

\section{Conclusions}

Through linear analysis tools, the controllability of different control structures for polymeric fuel cells has been compared. Results of the linear analysis indicate that different structures are suitable, what is confirmed through simulation. Several control structures are adequate if there is a tight operation around a nominal operating point, which is possible if the fuel cell is provided with a good electric power conditioning system. However, as has been seen through simulations, not all these structures are valid when the operating conditions move far from the nominal conditions. $\left(v_{\mathrm{cm}}-W_{\mathrm{an} \text {,in }}\right)$ and $\left(y_{\mathrm{O}_{2}, \text { ca,in }}-W_{\mathrm{an}, \text { in }}\right)$ control structures have the largest operating ranges. Of these two structures, $\left(y_{\mathrm{O}_{2}}\right.$,ca, in $\left.-W_{\mathrm{an} \text {,in }}\right)$ is less affected by the directionality of the system and has less

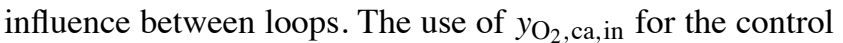
of $v_{\text {st }}$ has other important advantages that could justify the additional complexity required to change the oxidant composition. Simulations have also shown that, although a limitation on the $v_{\mathrm{st}}$ settling time is necessary, fuel cells can be controlled by linear decentralised feedback controllers with PI in each loop. Comparing the controllability indexes of a polymeric fuel cell at different operating conditions it has been found that a trade off between control performance and hydrogen consumption is possible. The controllability of the system can be improved changing the operating point.

\section{Acknowledgements}

This work has been funded partially by the projects CICYT DPI2002-03279 and CICYT DPI2004-06871-C02-01 of the Spanish Government.

\section{Appendix A}

The equations for the fuel cell voltage are:

$$
\begin{aligned}
E= & 1.229-8.5 \times 10^{-4}\left(T_{\mathrm{fc}}-298.15\right)+4.3085 \\
& \times 10^{-5} T_{\mathrm{fc}}\left[\ln \left(p_{\mathrm{H}_{2}}\right)+\frac{1}{2} \ln \left(P_{\mathrm{O}_{2}}\right)\right] \\
v_{\mathrm{act}}= & v_{0}+v_{\mathrm{a}}\left(1-e^{c_{1} i}\right) \\
v_{0}= & 0.279-8.5 \times 10^{-4}\left(T_{\mathrm{fc}}-298.15\right)+4.3085 \times 10^{-5} T_{\mathrm{fc}} \\
& \times\left[\ln \left(\frac{p_{\mathrm{ca}}-p_{\mathrm{sat}}}{1.01325}\right)+\frac{1}{2} \ln \left(\frac{0.1173\left(p_{\mathrm{ca}}-p_{\mathrm{sat}}\right)}{1.01325}\right)\right] \\
v_{\mathrm{a}}= & \left(-1.618 \times 10^{-5} T_{\mathrm{fc}}+1.618 \times 10^{-2}\right)\left(\frac{p_{\mathrm{O}_{2}}}{0.1173}+p_{\mathrm{sat}}\right)^{2} \\
& +\left(1.8 \times 10^{-4} T_{\mathrm{fc}}-0.166\right)\left(\frac{p_{\mathrm{O}_{2}}}{0.1173}+p_{\mathrm{sat}}\right) \\
& +\left(-5.8 \times 10^{-4} T_{\mathrm{fc}}+0.5736\right)
\end{aligned}
$$

$$
\begin{aligned}
& v_{\mathrm{ohm}}=i \times R_{\mathrm{ohm}} \\
& R_{\mathrm{ohm}}=\frac{t_{\mathrm{m}}}{\sigma_{\mathrm{m}}} \\
& \sigma_{\mathrm{m}}=\left(b_{11} \lambda_{\mathrm{m}}-b_{12}\right) \exp \left(b_{2}\left(\frac{1}{303}-\frac{1}{T_{\mathrm{fc}}}\right)\right) \\
& v_{\text {conc }}=i\left(c_{2} \frac{i}{i_{\text {max }}}\right)^{c_{3}}
\end{aligned}
$$

with the following values of the experimental parameters:

$$
c_{2}=\left\{\begin{array}{l}
\text { if } \frac{p_{\mathrm{O}_{2}}}{0.1173}+p_{\mathrm{sat}}<2, \\
\left(7.16 \times 10^{-4} T_{\mathrm{fc}}-0.622\right)\left(\frac{p_{\mathrm{O}_{2}}}{0.1173}+p_{\mathrm{sat}}\right) \\
\quad+\left(-1.45 \times 10^{-3} T_{\mathrm{fc}}+1.68\right) \text { else } \\
\left(8.66 \times 10^{-5} T_{\mathrm{fc}}-0.068\right)\left(\frac{p_{\mathrm{O}_{2}}}{0.1173}+p_{\mathrm{sat}}\right) \\
\quad+\left(-1.6 \times 10^{-4} T_{\mathrm{e}}+0.54\right)
\end{array}\right.
$$

$c_{1}=10$

$b_{11}=0.005139$

$$
b_{12}=0.00326
$$

$b_{2}=350$

$i_{\max }=2.2$

$c_{3}=2$ 
where $t_{\mathrm{m}}$ is the membrane thickness, and $\lambda_{\mathrm{m}}$ is the membrane water content.

\section{Appendix B}

Water transport across the membrane $W_{\mathrm{v}, \text { membr }}$ is

$$
\begin{aligned}
& W_{\mathrm{v}, \text { membr }}=M_{\mathrm{v}} A_{\mathrm{fc}} n\left(\frac{n_{\mathrm{d}} I_{\mathrm{st}}}{F}-D_{\mathrm{w}} \frac{c_{\mathrm{v}, \mathrm{ca}}-c_{\mathrm{v}, \text { an }}}{t_{\mathrm{m}}}\right) \\
& \left.a_{\mathrm{i}}=\frac{y_{\mathrm{v}, \mathrm{i}} p_{\mathrm{i}}}{p_{\mathrm{sat}, \mathrm{i}}}, \quad \mathrm{i}=\text { [anode, cathode }\right] \\
& \lambda_{\mathrm{m}}= \begin{cases}\text { if } 0<a_{\mathrm{i}} \leq 1, & 0.043+17.81 a_{\mathrm{i}}-39.85 a_{\mathrm{i}}^{2}+36 a_{\mathrm{i}}^{3} \\
\text { if } 1<a_{i} \leq 3, & 14+1.4\left(a_{\mathrm{i}}-1\right)\end{cases} \\
& n_{\mathrm{d}}=0.0029 \lambda_{\mathrm{m}}^{2}+0.05 \lambda_{\mathrm{m}}-3.4 \times 10^{-19} \\
& D_{\mathrm{w}}=D_{\lambda} \exp \left(2416\left(\frac{1}{303}-\frac{1}{T_{\mathrm{fc}}}\right)\right)
\end{aligned}
$$

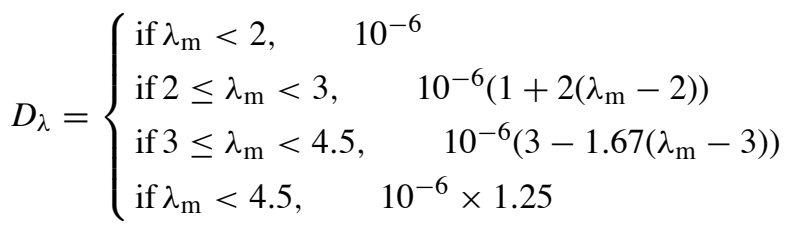

where $c_{\mathrm{v}, \mathrm{ca}}$ and $c_{\mathrm{v}, \text { an }}$ are the water concentrations in cathode and anode sides, and $y_{\mathrm{v}}$ is the molar fraction of water.

\section{References}

[1] G. Hoogers, in: G. Hoogers (Ed.), Fuel Cell Technology Handbook, CRC Press, 2003.

[2] J. Larminie, A. Dicks, Fuel Cell Systems Explained, John Wiley \& Sons Ltd., 2003.

[3] L. Carrette, K.A. Friedrich, U. Stimming, Fuel cells - fundamentals and applications, Fuel Cells 1 (2001) 1.

[4] J. Pukrushpan, A.G. Stefanopoulou, H. Peng, Control of fuel cell breathing, IEEE Control Syst. Mag., April 2004.

[5] K.Z. Yao, K. Karan, K.B. McAuley, P. Oosthuizen, B. Peppley, T. Xie, A review of mathematical models for hydrogen and direct methanol polymer electrolyte membrane fuel cells, Fuel cells 4 (2004) 1-2.

[6] J.M. Correa, F.A. Farret, L.N. Canha, An analysis of the dynamic performance of proton exchange membrane fuel cells using an electrochemical model. IECON'01: The 27th Annual Conference of the IEEE Industrial Electronic Society, 2001.

[7] J.T. Pukrushpan, H. Peng, A. Stefanopoulou, Simulation and analysis of transient fuel cell system performance based on a dynamic reactant flow model, ASME Int. Mechanical Engineering Congress \& Exposition, 2002.

[8] W. Yang, B. Bates, N. Fletcher, R. Pow, Control challenges and methodologies in fuel cell vehicle development, International Congress of Transportation Electronics 98C054, 1998.

[9] S. Yerramalla, A. Davari, Dynamic modelling and analysis of polymer electrolyte fuel cell, IEEE 2002.

[10] A. Simoglou, P. Argyropoulos, E.B. Martin, K. Scott, A.J. Morris, W.M. Taama, Dynamic modelling of the voltage response of direct methanol fuel cells and stacks Part 1: Model development and validation, Chem. Eng. Sci. 56 (2001) 67616772 .

[11] S. Skogestad, I. Postlethwaite, Multivariable Feedback Control, Analysis and Design, John Wiley \& Sons, 1996. 